\title{
Asymmetry of substantia nigra neuronal loss in Parkin- son's disease and its relevance to the mechanism of levodopa related motor fluctuations.
}

\author{
P A KEMPSTER, * W R G GIBB, $\dagger$ G M STERN,** A J LEES* $\dagger$ \\ From the Department of Neurology, Middlesex Hospital* and Department of Neuropathology, National Hospital \\ for Nervous Diseases, Maida Vale, $\dagger$ London, UK
}

\begin{abstract}
SUMMARY A pathological study of 21 patients with Parkinson's disease of asymmetrical onset revealed significant asymmetry of degeneration of the substantia nigra with greater neuronal loss contralateral to the initially affected body side. It has previously been suggested that decline in duration of effectiveness of levodopa doses in Parkinsonian patients with motor oscillations is caused by loss of nigro-striatal dopaminergic terminals with consequent reduction in striatal dopamine storage capacity. If this is true, duration of levodopa motor response should be shorter on the more severely affected body side in patients with asymmetrical disease, as loss of contralateral striatal dopamine storage capacity should be greater. Serial motor evaluations in 20 patients with asymmetrical Parkinson's disease failed to reveal any such asymmetry of duration of motor response to levodopa. It is suggested that striatal dopamine storage is not an important determinant of duration of clinical response to levodopa doses.
\end{abstract}

Oscillation of motor function is an almost inevitable occurrence after prolonged levodopa treatment for Parkinson's disease. Following oral doses of levodopa, the failure of smooth control of motor function has previously been described to take the form of a gradual deterioration from an initial "long duration" response of many hours to increasingly prominent "wearing off " effects after a few hours and finally to abrupt and seemingly random "on-off" oscillations.' It has been suggested that this is caused by progressive reduction in the amount of levodopa converted to dopamine and stored in nigro-striatal terminals. ${ }^{2}$ If this is so, residual dopamine storage capacity, in proportion to the number of surviving nigral neurons, should determine the duration of motor response to levodopa doses.

Asymmetry of clinical features is a common finding in Parkinson's disease. Many patients have unilateral motor deficits at the time of onset of symptoms. ${ }^{3}$ Bilateral motor involvement usually develops with increasing disease duration and most patients who

Address for reprint requests: Dr A J Lees, Institute of Neurology, The National Hospital for Nervous Diseases, Queen Square, London, WCIN 3BG, UK.

Received 6 May 1988 and in revised form 8 August 1988. Accepted 26 August 1988 have significant motor oscillations have more advanced disease. However, clinical asymmetry is still found late in the course of the disease, with more marked extrapyramidal involvement on the body side first affected. ${ }^{4}$ If degeneration of nigral neurons remains asymmetrical in the later stages of Parkinson's disease, then a mechanism of reduced conversion and storage of administered levodopa as a cause for declining duration of levodopa motor response would predict an earlier wearing off effect on the side initially affected.

The purpose of this study was to examine the pathology of Parkinson's disease with respect to asymmetry of nigral degeneration and to relate this to the time course of levodopa motor response in Parkinsonian patients with motor oscillations and clinical evidence of disease asymmetry.

\section{Patients and methods}

\section{Pathological study}

Brains from 21 patients ( 13 male and 8 female) who fulfilled the United Kingdom Parkinson's Disease Society Brain Bank criteria for Parkinson's disease ${ }^{5}$ were examined. In each case the diagnosis of Parkinson's disease was confirmed pathologically by the finding of nigral degeneration associated with numerous Lewy bodies. All had an asymmetrical onset of motor disability, with the initially involved body side recorded in the case notes. Cases were otherwise 
not selected for degree of asymmetry of extrapyramidal features. Mean age at death was $54 \cdot 1$ years and mean duration of disease was 11.8 years.

Brains had been fixed in $10 \%$ formol-saline for at least four weeks and selected areas were processed and examined. The midbrain was cut perpendicular to the axis of the brainstem through the emerging roots of the oculomotor nerves. This block was embedded in paraffin-wax and one or two $7 \mu \mathrm{m}$ thick sliced sections were stained with haematoxylin and eosin. The number of pigmented neurons and Lewy bodies in each half of the substantia nigra were counted directly with an eyepiece graticule at a magnification of $\times 400$. At the time of counting, the pattern of clinical disease asymmetry was unknown. For comparison, nigral neuron counts were performed on brains from 57 patients of similar age range with no known neurological disease and no detectable neuropathology.

Statistical analysis was by Student's $t$ test with comparison of paired samples.

\section{Clinical study}

Twenty patients (11 male and nine female) with clinically diagnosed Parkinson's disease and fuctuating motor response to levodopa medication were studied. Only patients who described an asymmetrical onset of motor symptoms, confirmed by reviewing medical records, and who had detectable clinical motor asymmetry in accordance with the side of onset were included. At the time of the study all these patients had bilateral abnormalities of motor function. Mean age was 57.3 years, mean duration of disease was 11.9 years and mean duration of levodopa treatment was $\mathbf{9 . 9}$ years.

Clinical asymmetry was quantified as follows: patients were assessed in "off" phases for asymmetry of hand bradykinesia, upper limb rigidity, limb tremor and arm swing when walking. In addition, involuntary limb movements during "on" periods were observed for asymmetry. Each patient was thus given a score out of five according to the number of these aspects of motor function that were objectively asymmetrical in keeping with the side of onset of extrapyramidal symptoms. Clinical details of all patients including motor asymmetry scores are summarised in the table.

Serial assessment of motor function was carried out for each patient following a single dose of levodopa $250 \mathrm{mg} /$ carbidopa $25 \mathrm{mg}$ while fasting. Unilateral hand tapping count was employed to measure upper limb bradykinesia ${ }^{6}$ and was presumed to reflect contralateral striatal motor response to levodopa. Subjects were asked to alternately tap two digital counters mounted $20 \mathrm{~cm}$ apart with one hand and the number of tap cycles completed in 30 second intervals was recorded. Tapping count for each hand was measured every fifteen minutes until motor function had returned to baseline level. In addition, the presence and severity of tremor and involuntary dyskinetic movements were recorded for each body side at similar intervals. Relative tapping speed between hands was also influenced by hand dominance, and did not mirror the degree of clinical motor asymmetry in all patients. All the patients had fluctuating motor function in relation to the levodopa/carbidopa dose detectable in both upper limbs by tapping counts.

\section{Results}

\section{Pathological study}

In every case, the substantia nigra showed moderate to severe loss of pigmented neurons. The amount of neuronal fragmentation, extraneural melanin and gliosis was similar between cases and between sides of the substantia nigra. Mean nigral neuron count was $222 \cdot 4$, SD 99 contralateral to the first affected body side and $289 \cdot 2$, SD 119 in ipsilateral substantia nigra ( $p$ $<0.02$ ). Results are represented graphically in fig 1 . Of the 21 cases examined, six showed a greater nigral cell number contralateral to the initially affected body side. Counts of neurons containing Lewy bodies were 9.3 per section contralateral and 9.4 per section ipsilateral to the first affected body side.

Mean unilateral nigral neuron counts in normal

Table Clinical details of patients

\begin{tabular}{|c|c|c|c|c|c|c|}
\hline Case Number & Age (years) & $\operatorname{Sex}$ & $\begin{array}{l}\text { Duration of disease } \\
\text { (years) }\end{array}$ & Side of onset & Motor asymmetry score & $\begin{array}{l}\text { Time to wearing off of motor } \\
\text { response (hours) }\end{array}$ \\
\hline $\begin{array}{r}1 \\
2 \\
3 \\
4 \\
5 \\
6 \\
7 \\
8 \\
9 \\
10 \\
11 \\
12 \\
13 \\
14 \\
15 \\
16 \\
17 \\
18 \\
19 \\
20\end{array}$ & $\begin{array}{l}39 \\
39 \\
72 \\
39 \\
43 \\
59 \\
69 \\
54 \\
67 \\
73 \\
43 \\
55 \\
69 \\
62 \\
61 \\
63 \\
73 \\
58 \\
58 \\
50\end{array}$ & $\begin{array}{l}\mathbf{F} \\
\mathbf{F} \\
\mathbf{M} \\
\mathbf{F} \\
\mathbf{F} \\
\mathbf{M} \\
\mathbf{M} \\
\mathbf{M} \\
\mathbf{F} \\
\mathbf{F} \\
\mathbf{M} \\
\mathbf{M} \\
\mathbf{M} \\
\mathbf{F} \\
\mathbf{F} \\
\mathbf{F} \\
\mathbf{M} \\
\mathbf{M} \\
\mathbf{M} \\
\mathbf{M}\end{array}$ & $\begin{array}{r}13 \\
4 \\
16 \\
17 \\
11 \\
15 \\
15 \\
13 \\
8 \\
8 \\
5 \\
10 \\
16 \\
17 \\
23 \\
10 \\
12 \\
6 \\
5 \\
15\end{array}$ & $\begin{array}{l}\text { Right } \\
\text { Right } \\
\text { Right } \\
\text { Left } \\
\text { Left } \\
\text { Left } \\
\text { Left } \\
\text { Right } \\
\text { Left } \\
\text { Left } \\
\text { Left } \\
\text { Left } \\
\text { Left } \\
\text { Left } \\
\text { Right } \\
\text { Left } \\
\text { Left } \\
\text { Left } \\
\text { Right } \\
\text { Right }\end{array}$ & $\begin{array}{l}2 \\
3 \\
2 \\
1 \\
1 \\
4 \\
1 \\
1 \\
2 \\
2 \\
5 \\
1 \\
2 \\
4 \\
1 \\
3 \\
3 \\
1 \\
1 \\
2 \\
2 \\
2 \\
1\end{array}$ & $\begin{array}{l}2 \cdot 25 \\
2 \cdot 25 \\
6 \cdot 25 \\
5 \cdot 5 \\
2 \cdot 0 \\
2 \cdot 75 \\
3 \cdot 25 \\
4 \cdot 5 \\
3 \cdot 5 \\
4 \cdot 25 \\
4 \cdot 25 \\
5 \cdot 75 \\
5 \cdot 0 \\
4 \cdot 5 \\
6 \cdot 0 \\
5 \cdot 5 \\
3 \cdot 0 \\
5 \cdot 0 \\
5 \cdot 5 \\
4 \cdot 25\end{array}$ \\
\hline
\end{tabular}




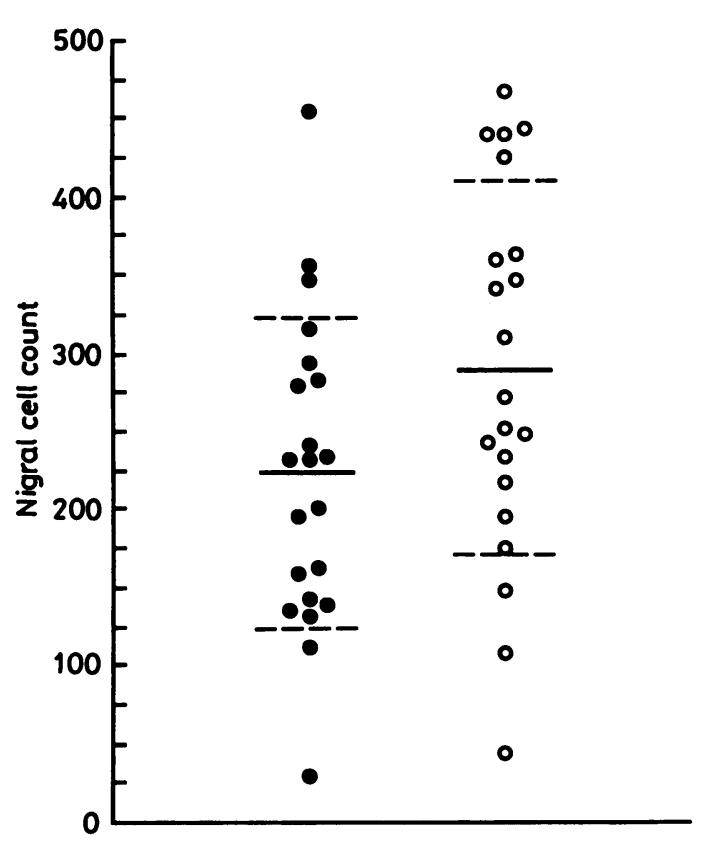

Fig 1 Graph of neuron counts in substantia nigra contralateral (solid circles) and ipsilateral (open circles) to first affected body side/Mean counts and one standard deviation are shown.

control cases were as follows: age 40-49 years: 1042, SD 148 neurons age 50-59 years: 963 , SD 194 neurons age 60-69 years: 864 , SD 197 neurons age 70-79 years: 850 , SD 237 neurons

\section{Clinical study}

Time from levodopa/carbodopa dose until waning of motor response ranged from 2 to 6.5 hours. In no case was was there evidence of earlier wearing off of motor response on the initially and worst affected body side. A typical profiile of levodopa motor response is shown in fig 2. In most cases, the wearing off phase spanned several tapping count measurements, making it unlikely that asynchronous wearing off was missed by motor assessments at 15 minute intervals.

Two patients showed conspicuous motor asymmetry during wearing off phases with apparent prolongation of improved motor function on the worst affected body side. This side showed transiently better tapping counts and persistence of dyskinetic movements at a time when contralateral limb tremor had returned. However, the pattern of serial measurements (fig 3 ) revealed that the onset of declining motor function was simultaneous for both body sides.

\section{Discussion}

The pathological studies suggest that patients with Parkinson's disease of asymmetrical onset may have significant asymmetry of the number of surviving nigral neurons late in the disease course. Asymmetry was of the order of a $25 \%$ reduction in the substantia nigra contralateral to the initially more affected body side with respect to the less affected side. Despite this significant difference, six out of 21 brains examined did not show the expected asymmetry. The clinical information available on pathologically examined cases did not allow quantitation of degree of motor asymmetry and this may have been mild in some patients. Alternatively, minor errors in cutting perpendicular midbrain sections could have led to comparison of different nigral regions between sides and masking of asymmetrical nigral cell loss. Counting of nigral neurons in whole midbrain sections by this technique may also underestimate the degree of degeneration of cells projecting to the ipsilateral putamen. Striatal dopamine deficiency is most severe in the putamen in Parkinson's disease ${ }^{7}$ and animal neurophysiological studies suggest that the putamen is of major importance in mediating the motor functions of the basal ganglia. ${ }^{8}$ Cell loss in the substantia nigra is not uniform, preferentially affecting cellular regions with synaptic connections in the putamen. ${ }^{9}$ Thus the degree of asymmetry in these regions of the substantia nigra may be greater than is suggested by whole nigral neuron counts. Neuro-imaging studies using labelled levodopa confirm that striatal neurochemical asym-

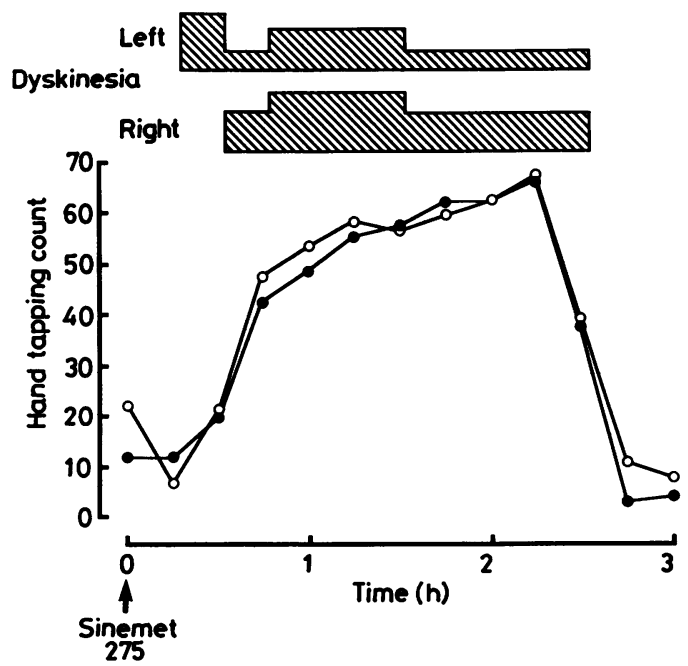

Fig 2 Serial motor assessments following oral levodopa 250 $\mathrm{mg} /$ carbidopa $25 \mathrm{mg}$ dose in Case 1 . Onset of motor symptoms was right sided. Hand tapping counts are shown for right (closed circles) and left (open circles) hands. 


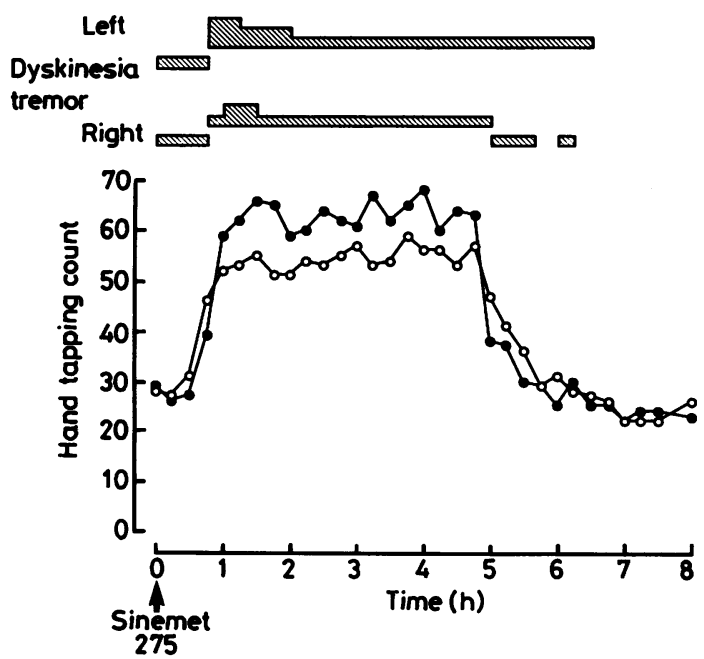

Fig 3 Serial motor assessments in Case 12 following levodopa/carbidopa dose. Left sided onset of disease. Wearing off of motor response begins simultaneously for right (closed circles) and left (open circles) hands five hours later according to tapping counts. However, left hand tapping count initially declines more slowly and right limb tremor recurs while left limb dyskinetic movements persist.

metry occurs in advanced Parkinson's disease. Significant asymmetry of putaminal accumulation of radio-isotope label is seen in patients who retain some clinical asymmetry despite development of bilateral motor deficits with progression of disease. ${ }^{10}$

It was assumed that at least a similar degree of nigral asymmetry was present in the clinically examined patient group with asymmetrical motor function. The presence in all patients of an unequivocal response to levodopa and absence of signs of non-extrapyramidal neurological dysfunction suggested that the clinical and pathological patient groups were matched for underlying pathology of nigral degeneration associated with Lewy bodies. Clinically and pathologically examined patients were also well matched for age and duration of disease.

Time from ingestion of a single dose of levodopa/ carbidopa to wearing off of motor response varied from 2 to 6.5 hours. Duration of motor response did not correlate closely with duration or severity of Parkinson's disease. Fluctuation of motor function in relation to levodopa doses in the patients that were studied ranged from mildly symptomatic oscillations to severe "on-off" effects with marked motor disability during "off" periods. However, no patient showed evidence that wearing off occurred on the more affected body side before the less affeeted side.- If duration of response to administered levodopa is dependant on storage of dopamine in surviving nigro- striatal terminals, one would expect to detect some asymmetry of duration of motor response as storage capacity should be less in the striatum contralateral to the worst and initially affected body side.

Reduction in striatal dopamine storage capacity in Parkinsonian patients is suggested by positron emission tomographic studies. In patients with advanced Parkinson's disease, radio-isotope labelled levodopa accumulates in the striatum for a shorter time in comparison to normal controls. " This corresponds to a reduced number of dopaminergic terminals but does not necessarily imply that the dopamine storage capacity in the individual surviving terminals is reduced. Furthermore, the influence of levodopa on the pattern of synaptic activity in these surviving terminals cannot readily be inferred from such neuroimaging data. Motor response to administered levodopa may not be dependant on neurotransmission by nigro-striatal neurons. Aromatic amino acid decarboxylase, which catalyses the conversion of levodopa to dopamine, is quite widely distributed in brain tissue and animal studies suggest that significant levodopa conversion may occur outside aminergic neurons. ${ }^{1213}$ If levodopa medication results in significant striatal dopamine receptor stimulation by dopamine which is not synthesised, stored and released by nigro-striatal cells, reduced capacity for the nigro-striatal neurons to store dopamine would not be of importance in determining the time course of levodopa motor responses.

In Parkinsonian patients with presumed asymmetry of nigral cell degeneration, the failure to detect significant asymmetry in duration of motor response to levodopa argues against its dependence on storage of dopamine in nigro-striatal neurons. It is suggested that the nigro-striatal neuronal dopamine storage hypothesis does not explain the differences in duration of effectiveness of levodopa doses among patients with Parkinson's disease.

PAK was supported by the Kate Stillman Research Fellowship and WRGG was supported by a grant from the Medical Research Council.

\section{References}

1 Marsden CD, Parkes JD, Quinn N. Fluctuations in disability in Parkinson's disease-clinical aspects. In: Marsden CD, Fahn S, eds. Movement Disorders. London: Butterworths 1981:91-122.

2 Marsden CD, "On-off" phenomenon in Parkinson's disease. In: Rinne UK, Klinger M, Stamm G, eds. Parkinson's Disease: Current Progress, Problems in Management. Amsterdam: Elsevier, 1980:241-54.

3 Hoehn MM, Yahr MD. Parkinsonism: onset, progression and mortality. Neurology 1967;17:427-42.

4 Fross RD, Tsin JKC, McLennan DJ, Schultzer M, Calne DB. Asymmetry in Parkinson's disease. Neurology 
1987;37 (suppl):320.

5 Rogers D, Lees AJ, Smith E, Trimble M, Stern GM. Bradyphrenia in Parkinson's disease and psychomotor retardation in depressive illness. Brain 1987;110: 761-76.

6 Nutt JG, Woodward WR, Hammerstaad JP, Carter JH, Anderson JL. The "on-off" phenomenon in Parkinson's disease: relation to levodopa absorption and transport. N Engl J Med 1984;310:483-8.

$7 \mathrm{Kish}$ SJ, Shannak K, Hornykiewicz O. Uneven pattern of dopamine loss in the striatum of patients with idiopathic Parkinson's disease. $N$ Engl J Med 1988;318: 876-80.

8 Crutcher MD, DeLong MR. Single cell studies in the primate putamen. I. Functional organization. Exp Brain Res 1984;53:233-43.

9 Hassler R. Zur Pathologie der Paralysis agitans und der postenzephalitischen Parkinsonismus. $J$ Psychol Neurol 1938;48:387-476.

10 Martin WRW, Calne DB. Imaging techniques and movement disorders. In: Marsden CD, Fahn, S, eds. Movement Disorders 2. London: Butterworths 1987: 4-16.

11 Leenders KL, Palmer AJ, Quinn N, et al. Brain dopamine metabolism in patients with Parkinson's disease measured with positron emission tomography. $J$ Neurol Neurosurg Psychiatry 1986;49:853-60.

12 Melamed E, Hefti F, Wurtman RJ. Non-aminergic neurons convert exogenous L-dopa to dopamine in Parkinsonism. Ann Neurol 1980;8:558-63.

13 Melamed E, Hefti F, Pettibone DJ, Liebman J, Wurtman RJ. Localisation of aromatic L-amino acid decarboxylase in rat corpus striatum: implications for action of $L$ dopa in Parkinsonism. Neurology 1981;31:651-5. 